\title{
Women's Perspectives on Contraceptive-Induced Amenorrhea in Burkina Faso and Uganda
}

\begin{abstract}
CONTEXT: Women's concerns about contraceptive-induced menstrual changes can lead to method discontinuation and nonuse, contributing to unmet need for contraception. Research on women's perceptions of amenorrhea related to longer acting methods and in low-income countries is limited.
\end{abstract}

\begin{abstract}
METHODS: Data were from nationally representative household surveys and focus group discussions with women of reproductive age conducted in Burkina Faso and Uganda in 2016-2017. Bivariate cross-tabulations and multivariate logistic regression analyses were used to examine sociodemographic and reproductive characteristics associated with women's attitudes about contraceptive-induced amenorrhea ( $n=2,673$ for Burkina Faso and 2,281 for Uganda); menstrual health determinants were also examined for Burkina Faso. Qualitative data from focus group discussions were analyzed to understand reasons behind women's attitudes and how they influence contraceptive decision making.

RESULTS: Sixty-five percent of women in Burkina Faso and 40\% in Uganda reported they would choose a method that caused amenorrhea during use. In Burkina Faso, the predicted probability of accepting amenorrhea was higher for women aged 15-19 (compared with older women), living in rural areas, married and cohabiting (compared with never married), currently using a contraceptive method (compared with never users) and from Mossi households (compared with Gourmantché); menstrual health practices were not associated with amenorrhea acceptability. In Uganda, the least wealthy women had the highest predicted probability of accepting amenorrhea (51\%). Qualitative analysis revealed a variety of reasons for women's attitudes about amenorrhea and differences by country, but the relationship between these attitudes and contraceptive decision making was similar across countries.
\end{abstract}

CONCLUSIONS: Addressing misconceptions about contraception and menstruation may result in more informed method decision making.

International Perspectives on Sexual and Reproductive Health, 2020,46:247-262; doi: https://doi.org/10.1363/46e1520

Women's concerns about real and perceived contraceptive side effects or other health effects of contraceptive use are a key contributor to unmet need for family planning. Demographic and Health Survey data from Sub-Saharan Africa show such concerns are the most frequently cited reason for contraceptive nonuse among married women with an unmet need. ${ }^{1-3}$ Side effects and health concerns are also the most or second-most frequently cited reason for contraceptive discontinuation in more than $90 \%$ of countries in Sub-Saharan Africa. ${ }^{4}$

One type of side effect women can experience when using contraceptive methods-including the pill, injectable, implant and IUD-is changes to their menstrual cycle, and with hormonal methods, women may experience amenorrhea or a pause in menstrual bleeding. ${ }^{5-8}$ As recently reviewed by Polis et al., these contraceptiveinduced menstrual changes-including amenorrhea-can cause women to discontinue or not use methods. ${ }^{9}$

Recent research on contraceptive-induced amenorrhea has almost exclusively focused on women in middle- and high-income countries, and has largely examined amenorrhea in the context of continuous use of the pill (i.e., skipping the hormone-free placebo pills within a monthly pack of combined oral contraceptives). ${ }^{9-16}$ The recent review by Polis et al. discusses this research in detail. ${ }^{9}$ To summarize, views about contraceptive-induced amenorrhea are often related to how women and communities view menstruation more broadly, and women's concerns can stem from fears of subsequent health effects of amenorrhea, particularly infertility. 10,13,14,17,18 Some women see amenorrhea as unnatural and worry about blood accumulating inside their bodies if they are not menstruating. 10,14,17 Because menstrual blood is often viewed as "dirty" or "poison," and menstruation is perceived as a way to cleanse the body, the fear of this accumulation is often worrisome to women and seen as a threat to their health. ${ }^{13,14,17,19,20}$ In addition, because most women who practice contraception do so to prevent pregnancy, the absence of bleeding can be concerning if taken as a sign of pregnancy, especially for those without easy access to pregnancy testing. ${ }^{13,14}$

Some of this research on contraceptive-induced amenorrhea has examined the relationship between amenorrhea acceptability and various sociodemographic and reproductive characteristics. Studies have found age, education, parity and fertility intentions to be associated with amenorrhea acceptability, but results have been
By Amelia C.L. Mackenzie, Siân L. Curtis, Rebecca L. Callahan,

Elizabeth $E$.

Tolley, llene S. Speizer, Sandra L. Martin and Aurélie Brunie

At the time the study was conducted, Amelia Mackenzie was a doctoral candidate, Department of Maternal and Child Health, Gillings School of Global Public Health, University of North Carolina at Chapel Hill, NC, USA. Siân Curtis is associate professor, Ilene Speizer is professor, and Sandra Martin is professor and associate chair for research-all in the Department of Maternal and Child Health, Gillings School of Global Public Health.

Rebecca Callahan is associate director, Department of Contraceptive Technology Innovation; Elizabeth Tolley is director, Behavioral, Epidemiological and Clinical Sciences Division; and Aurélie Brunie is scientist, Health Services Research Divisionall with FHI 360, Durham, NC, USA. 
inconsistent. ${ }^{11,14,17,20,21}$ A concern about the financial cost of menstrual products may also make women more willing to accept contraceptive-induced amenorrhea. ${ }^{18,22}$ In addition, some standard menstrual bleeding patterns and symptoms experienced prior to contraceptive use (e.g., pain from menstrual cramping, longer or higher volume bleeding, and stress) may make women more likely to accept amenorrhea. ${ }^{15,16,20,22,23}$

Overall, the literature on the acceptability of amenorrhea while using a contraceptive is not conclusive, and the research is limited by its focus on middle- and high-income countries, and on continuous use of the pill. ${ }^{9}$ Therefore, to address these specific gaps in the literature, this study aimed to examine women's perspectives on amenorrhea associated with use of any contraceptive method in two Sub-Saharan African countries: Burkina Faso and Uganda. We used household survey data and focus group discussions (FGDs) to examine factors associated with women's attitudes about amenorrhea, women's reasons for those attitudes and how they influence women's contraceptive decision making.

\section{METHODS}

Data for this analysis were from a cross-sectional, sequential mixed-methods study conducted by FHI 360 in Burkina Faso and Uganda to understand the needs, preferences and perspectives of potential contraceptive users, providers, program implementers and policy makers to inform the development of six new long-acting contraceptive methods. ${ }^{24,25}$ The FHI 360 study was conducted in partnership with the Institut Supérieur des Sciences de la Population of the University of Ouagadougou in Burkina Faso, Makerere University School of Public Health in Uganda, and Performance Monitoring and Accountability 2020 (PMA2020) at the Bill \& Melinda Gates Institute for Population and Reproductive Health in the Johns Hopkins Bloomberg School of Public Health. It received approval by the Protection of Human Subjects Committee at FHI 360; the Comité d'Ethique pour la Recherche en Santé in Burkina Faso; and the Makerere University School of Public Health Higher Degrees Research and Ethics Committee and the National Council for Science and Technology in Uganda. This secondary analysis was exempted from institutional review board review by the Office of Human Research Ethics at the University of North Carolina at Chapel Hill.

\section{Study Countries}

Burkina Faso and Uganda were selected because of their PMA2020 programs and differing geographic, sociocultural and family planning contexts. At the time of data collection, among all women aged 15-49 in both countries, contraceptive use was low (23\% in Burkina Faso and $31 \%$ in Uganda), unmet need for family planning was high (24\% each), and unintended pregnancy was notable ( $32 \%$ in Burkina Faso and 44\% in Uganda) ${ }^{26,27}$ However, the two countries differed in terms of method mix and service delivery context. In Burkina Faso, the three most commonly used methods were the implant, injectable and pill (43\%, 30\% and 11\%, respectively), while in Uganda, they were the injectable, implant and traditional methods (47\%, 13\% and 12\%). ${ }^{26,27}$ The proportion of all current modern contraceptive users who received their method from public facilities was $87 \%$ in Burkina Faso and 60\% in Uganda, and the proportion who paid for family planning services was 66\% in Burkina Faso and 42\% in Uganda. ${ }^{28,29}$ All public facilities sampled in Burkina Faso offered at least five contraceptive methods, compared with only $42 \%$ in Uganda. ${ }^{28,29}$ Furthermore, in Burkina Faso, community health workers only provided condoms and resupplied the pill, whereas in Uganda, community health workers could provide condoms, the pill and the injectable.

Access to safe abortion is also low in both countries. In Burkina Faso, abortion is legally permitted to save the life and health of the woman, and in cases of rape, incest or severe fetal abnormality; however, knowledge of these laws is low, and most abortions are performed in secret-often under unsafe conditions. ${ }^{30}$ In Uganda, abortion is legally allowed only to save the life of the woman, although national guidelines additionally permit it in cases of rape, incest or severe fetal abnormality, as well as when the pregnant women is HIV-positive. As in Burkina Faso, however, Uganda's laws and policies regarding abortion are often inconsistently interpreted, resulting in limited access to safe abortion services. ${ }^{31}$ The abortion rate was estimated at 25 per 1,000 women aged 15-49 in Burkina Faso in 2012 and 39 per 1,000 in Uganda in 2013, which are both similar to regional estimates. ${ }^{30-32}$

\section{Quantitative Analysis}

- Data. Quantitative data came from nationally representative PMA2020 household surveys. Data were collected between November 2016 and January 2017 in Burkina Faso (Round 4), and between April and May 2016 in Uganda (Round 4). In each country, a probability sample of households was selected using a two-stage cluster design-with urban-rural strata in Burkina Faso, and urban-rural and subregion strata in Uganda. ${ }^{26,27}$ In each selected household, a household survey was conducted, and all women aged 15-49 who were de facto residents of the household (i.e., stayed there the night before the survey) were eligible for a women's questionnaire. Among eligible women, potential contraceptive users (i.e., those not using a permanent contraceptive method who stated they would consider using a newly developed contraceptive method in the future and, in Burkina Faso, stated they thought they would use any contraceptive method in the future) were eligible for a module of 12 questions added by FHI 360 to the end of the women's questionnaire.

The proportion of women who completed the PMA2020 women's questionnaire who also completed the added module of questions was $86 \%$ in Burkina Faso $(n=2,743)$ and $63 \%$ in Uganda $(n=2,403)$, which was $99.8 \%$ and $100 \%$ of women who were eligible for the 
module, respectively. Women who were younger, had a higher parity, wanted more children or were pregnant had a significantly higher predicted probability of being eligible for the study questions in both countries; in Burkina Faso, this was also true for women in rural areas and by household ethnicity (not shown).

- Measures. The added module included questions asking women about how various method attributes related to their contraceptive decision making and about their interest in using six methods currently under development. One question asked women their attitude about amenorrhea: "With some contraceptive methods, women do not get their period, but their period and their fertility return when they stop using it. Would you choose a method that stops your period?" For our dependent variable, we considered women who responded "yes" to find amenorrhea to be acceptable or desirable, which we refer to broadly as amenorrhea acceptability. We note, however, that although the clinical definition of amenorrhea is typically 90 days without any menstrual bleeding, the study question did not specify the duration of bleeding absence. This specific distinction is likely not particularly meaningful to women, especially in the context of contraceptive-induced amenorrhea as compared with pathological amenorrhea. In addition, there is evidence women and providers interpret amenorrhea and its implications differently. ${ }^{18,33}$

In Burkina Faso, the PMA2020 survey also collected data on menstrual health practices among women who menstruated in the last 90 days and were not pregnant (67\% of women who completed the added module of questions; $\mathrm{n}=1,847$ ). Menstrual health questions used in our analysis asked women about materials they used to collect or absorb menstrual blood during their last period and about sanitation facilities or other places they changed, washed, dried or disposed of these materials. In addition, women were asked if there was anything else they needed to better manage their menstruation (i.e., any unmet menstrual health needs).

- Analysis. To examine sociodemographic and reproductive characteristics associated with attitudes about amenorrhea in each country, we used bivariate cross-tabulations with design-adjusted Rao-Scott tests and multivariate logistic regression. Our models included categorical variables for sociodemographic characteristics: age-group, urbanrural residence, education (primary or less, or secondary or more), wealth (in terciles for Burkina Faso and quintiles for Uganda), and marital status (never married, married and cohabiting, married and not cohabiting, and previously married). Categorical variables were also included for reproductive characteristics: parity $(0,1,2-4,5-7, \geq 8$ children in Burkina Faso and 0, 1, 2-3, 4-5, $\geq 6$ in Uganda), fertility intentions (whether the women wanted more children or not), contraceptive use (never, past or current use of any contraceptive method), sexual activity in the last month (yes or no), and pregnancy status (yes or no/don't know). The model for Burkina Faso included additional sociodemographic characteristics (i.e., language in which the women's questionnaire was conducted, and religion and ethnicity of the head of the women's household), because these data were only collected in Burkina Faso. The analysis sample of women with nonmissing values for all covariates was 2,673 in Burkina Faso (97\% of women who completed the added module of questions) and 2,281 in Uganda (95\%); all covariates had less than 1\% missingness except for sexual activity, which was $1.5 \%$ in Burkina Faso and 3.4\% in Uganda). We fit a second multivariate model for Burkina Faso of recently menstruating women to identify menstrual health practices associated with women's attitudes about amenorrhea. The analysis sample for this second model was 1,834 women (99\%).

For all multivariate models, all covariates were included, regardless of statistical significance in the bivariate crosstabulations. We used Stata 14, and all analyses were adjusted for the complex sampling design and unit nonresponse to the women's questionnaire using svy commands with sampling variables and weights provided with the data set, and methods appropriate for subpopulation analysis (e.g., subpop option). We considered p-values below an alpha of 0.05 to be statistically significant.

\section{Qualitative Analysis}

- Data. Qualitative data came from FGDs with women selected purposively, and stratified by in-country region and current contraceptive use, to ensure the sample captured a range of different contraceptive experiences. FGDs were conducted between April and May 2016 in Burkina Faso, and in February 2016 in Uganda. In Burkina Faso, 16 FGDs with a total of 132 women were conducted in five of the country's 13 regions (Boucle du Mouhoun, East, North, Ouagadougou, and Southwest); six were with current contraceptive users and 10 were with current nonusers. In Uganda, 30 FGDs with a total of 239 women were conducted in each of the country's four regions plus the capital city (Central, Eastern, Kampala, Northern, and Western); 10 were with current users of long-acting reversible contraceptives (i.e., the implant and IUD; LARCs), 10 were with current users of short-acting hormonal methods (i.e., the pill and injectable) and 10 were with current nonusers. Within regions, FGDs were conducted in PMA2020 enumeration areas.

Women were eligible for the qualitative study if they were aged 18-49, or aged 15-17 and married. Women opposed to family planning or not currently practicing contraception because they were trying to get pregnant were excluded, as they were not in the target population of interest for the FHI 360 study. Participants were identified through providers at local health centers in Burkina Faso and largely by community health workers in Uganda. FGDs were conducted using semi-structured discussion guides in local languages by trained moderators, with an audio recording and notes taken during the discussion; additional details are published elsewhere..$^{24,25}$ Sociodemographic and reproductive information about FGD participants was also collected. FGD moderators and 
notetakers translated and transcribed audio recordings of the FGDs into French in Burkina Faso and into English in Uganda.

-Analysis. For this secondary analysis, we used a section of the discussion guide that asked women about past and current contraceptive experiences-and specifically about how much of a problem it would be if a method caused amenorrhea. As with the quantitative data, a specific duration of bleeding absence for amenorrhea was not specified in the guide. We used NVivo 11 and Excel for qualitative analysis.

A team of four coders independently coded FGD transcripts for the FHI 360 study using a codebook created with deductive codes from the FGD guides and inductive codes that emerged during analysis. We used portions of the transcripts coded as related to contraceptive-induced menstrual changes for this secondary analysis, with transcript portions from Burkina Faso translated from French into English. The first author developed an initial codebook of contraceptive-induced menstrual change subcodes that was iteratively updated while reviewing the transcripts and then used for subcoding. For each FGD, data on every coded mention of amenorrhea was extracted into a detailed matrix evaluating three parameters dictated by our research question: attitudes about amenorrhea, the reasons for these attitudes and any impact on contraceptive decision making. The components and categories of these parameters were developed iteratively during the analysis process.

Based on specific terminology used in FGDs, we grouped attitudes about amenorrhea into three categories: unacceptable (e.g., a problem, concern, not good), acceptable (e.g., could manage it, not a problem, did not fear it) or desirable (e.g., good, would welcome it, would be a relief or calming). If the discussion did not indicate an explicit attitude with specific terminology, attitude was not inferred, and it was categorized as not stated.

The reasons discussed in FGDs for participants' amenorrhea attitudes were grouped into themes developed iteratively during transcript analysis (e.g., relating to health impacts of amenorrhea, provider counseling about amenorrhea, partner's concerns about amenorrhea). The broad theme of health impacts was categorized into subtopics given their distinct nature (e.g., aches or pains, sexual effects, strength or energy changes). Not all discussion of amenorrhea attitudes in FGDs cited a reason. Because most reasons could be viewed both positively and negatively, they were not explicitly divided as such. For example, weight gain could be seen as a reason for desiring amenorrhea or finding it unacceptable.

We categorized the various impacts of attitudes toward amenorrhea on contraceptive decision making discussed in the FGDs into three risk categories-low, medium and high-according to the level of risk of unintended pregnancy to which participants might be exposed from that contraceptive behavior. Low-risk contraceptive decision making included continuation or willingness to use if or when experiencing amenorrhea, or not viewing amenorrhea as a barrier to use. Medium-risk contraceptive decision making included switching methods, considering discontinuing or switching, temporarily pausing method use, or choosing a less effective method if or when experiencing amenorrhea. High-risk contraceptive decision making included nonuse, discontinuation, or unwillingness to use if or when experiencing amenorrhea, or viewing amenorrhea as a barrier to use. Not all discussion of amenorrhea attitudes in FGDs cited an impact on contraceptive decision making.

When an FGD had a participant who discussed more than one attitude about amenorrhea, the corresponding reasons and any impacts on contraceptive decision making were attributed to each attitude separately. For example, a participant might state she liked amenorrhea because she usually experiences painful menstrual cramping, but she worried her bleeding might become high in volume when amenorrhea ended and this worry would prevent her from using an amenorrhea-inducing method. In such a case, the participant's desirable attitude toward amenorrhea would be attributed to its alleviation of her standard bleeding and no stated impact on contraceptive decision making, and her unacceptable attitude would be attributed to her concern about problems when bleeding resumed and highrisk contraceptive nonuse.

\section{RESULTS}

\section{Quantitative Findings}

- Descriptive and bivariate results. In both countries, about $60 \%$ of women in our samples were younger than 30 (59\% in Burkina Faso and 64\% in Uganda; Table 1), three-quarters were currently married (76\% and $73 \%$, respectively), and more than three-quarters had at least one child (78\% and $82 \%$ ). About $80 \%$ in both countries lived in rural areas (76\% and $83 \%$ ), but a greater proportion of women in Uganda than in Burkina Faso had attended secondary school (30\% vs. $20 \%$ ). In addition, a greater proportion of women in Uganda had ever used a contraceptive method (62\% vs. $44 \%$ ). In Burkina Faso, Mossi ethnicity and Mooré language were the plurality (43\% and 47\%, respectively), and 63\% of women were Muslim; data on ethnicity, language and religion were not collected in Uganda.

Sixty-five percent of women in Burkina Faso and 40\% in Uganda indicated they would choose a method that caused amenorrhea during use. In bivariate analyses, amenorrhea acceptability was higher among women in rural areas than in urban areas ( $72 \%$ vs. $42 \%$, respectively, in Burkina Faso; and $43 \%$ vs. $27 \%$ in Uganda), among women with a primary or no education than those with a secondary or higher education ( $68 \%$ vs. $52 \%$, and $43 \%$ vs. $32 \%$ ), and among those who were currently married than those never married (66-69\% vs. 50\%, and $41-43 \%$ vs. $33 \%$ ). Also, in both countries, amenorrhea acceptability increased as wealth decreased and as parity increased. In addition, in Burkina Faso, amenorrhea acceptability was higher among women who were sexually active in the last 
TABLE 1. Percentage distribution of women aged 15-49 who were potential contraceptive users; and percentage of those who would choose a method that caused amenorrhea during use-both by sociodemographic and reproductive health characteristics, according to country, PMA2020 survey, Burkina Faso 20162017 and Uganda 2016

\begin{tabular}{|c|c|c|c|c|}
\hline \multirow[b]{2}{*}{ Characteristic } & \multicolumn{2}{|c|}{ Burkina Faso } & \multicolumn{2}{|c|}{ Uganda } \\
\hline & $\begin{array}{c}\% \\
(n=2,673)\end{array}$ & $\begin{array}{l}\text { \% amenorrhea } \\
\text { acceptable } \\
(n=1,528)\end{array}$ & $\begin{array}{c}\% \\
(n=2,281)\end{array}$ & $\begin{array}{c}\text { \% amenorrhea } \\
\text { acceptable } \\
(\mathrm{n}=880)\end{array}$ \\
\hline \multicolumn{5}{|l|}{ Age } \\
\hline $15-19$ & 21.6 & 63.0 & 19.1 & 38.0 \\
\hline $20-24$ & 18.9 & 63.5 & 25.6 & 39.1 \\
\hline $25-29$ & 18.9 & 66.8 & 19.5 & 36.1 \\
\hline $30-34$ & 14.8 & 68.5 & 16.6 & 45.9 \\
\hline $35-49$ & 25.7 & 63.8 & 19.3 & 41.6 \\
\hline \multicolumn{2}{|l|}{ Residence } & $* * *$ & & ** \\
\hline Urban & 23.8 & 42.0 & 17.2 & 27.2 \\
\hline Rural & 76.2 & 72.0 & 82.8 & 42.6 \\
\hline \multirow{3}{*}{ 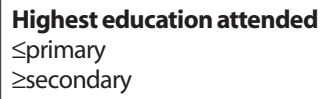 } & & $* * *$ & & ${ }^{* *}$ \\
\hline & 79.9 & 68.0 & 70.4 & 43.3 \\
\hline & 20.1 & 52.3 & 29.6 & 31.8 \\
\hline \multicolumn{2}{|l|}{ Wealth index $\dagger$} & $* * *$ & & $* * *$ \\
\hline Lowest & 34.0 & 68.8 & 19.3 & 52.7 \\
\hline Second & na & na & 18.8 & 44.8 \\
\hline Middle & 32.2 & 73.0 & 21.2 & 41.0 \\
\hline Fourth & na & na & 20.5 & 37.7 \\
\hline Highest & 33.8 & 53.1 & 20.2 & 24.1 \\
\hline \multicolumn{2}{|l|}{ Marital status } & $* * *$ & & * \\
\hline Never married & 20.1 & 50.4 & 17.9 & 33.1 \\
\hline Married and cohabiting & 67.0 & 69.1 & 61.0 & 42.5 \\
\hline Married and not cohabiting & 9.2 & 66.2 & 11.6 & 41.3 \\
\hline Previously married & 3.7 & 63.0 & 9.5 & 34.1 \\
\hline \multicolumn{2}{|l|}{ Parity $\neq$} & $* * *$ & & $* * *$ \\
\hline 0 & 22.1 & 53.3 & 18.3 & 33.9 \\
\hline 1 & 13.4 & 65.0 & 17.1 & 32.2 \\
\hline $2-4 / 2-3$ & 35.5 & 70.1 & 26.2 & 40.9 \\
\hline $5-7 / 4-5$ & 21.4 & 65.8 & 19.4 & 44.4 \\
\hline$\geq 8 / \geq 6$ & 7.5 & 70.9 & 19.1 & 46.6 \\
\hline \multicolumn{5}{|l|}{ Wants more children } \\
\hline Yes & 17.9 & 64.0 & 28.6 & 41.2 \\
\hline No & 82.1 & 65.0 & 71.4 & 39.4 \\
\hline \multicolumn{5}{|l|}{ Any contraceptive use } \\
\hline Never-user & 55.6 & 65.0 & 38.0 & 37.6 \\
\hline Past user & 17.7 & 62.2 & 25.7 & 40.7 \\
\hline Current user & 26.6 & 66.3 & 36.3 & 41.8 \\
\hline \multicolumn{2}{|l|}{ Sexually active in last month } & * & & \\
\hline No & 41.9 & 61.2 & 32.1 & 37.2 \\
\hline Yes & 58.1 & 67.5 & 67.9 & 41.2 \\
\hline \multicolumn{2}{|l|}{ Pregnant } & ** & & \\
\hline No/don't know & 90.6 & 64.0 & 86.6 & 39.7 \\
\hline Yes & 9.4 & 72.9 & 13.4 & 41.5 \\
\hline \multicolumn{2}{|l|}{ Survey language } & ** & & \\
\hline Mooré & 42.3 & 66.3 & na & na \\
\hline French & 11.0 & 39.3 & na & na \\
\hline Other & 46.7 & 69.5 & na & na \\
\hline \multicolumn{5}{|l|}{ Head of household's religion } \\
\hline Muslim & 62.1 & 64.7 & na & na \\
\hline Other & 37.9 & 65.0 & na & na \\
\hline \multicolumn{5}{|l|}{ Head of household's ethnicity } \\
\hline Mossi & 46.4 & 65.8 & na & na \\
\hline Gourmantché & 12.5 & 64.3 & na & na \\
\hline Fulfuldé/Peulh/Touareg/Bella & 7.1 & 66.3 & na & na \\
\hline Dioula/Bobo/Senoufu/Other & 11.1 & 56.4 & na & na \\
\hline Other Burkinabè & 22.9 & 66.7 & na & na \\
\hline Total & 100.0 & 64.8 & 100.0 & 39.9 \\
\hline
\end{tabular}

${ }^{*} \mathrm{p} \leq .05 .{ }^{* *} \mathrm{p} \leq .01 .{ }^{* * *} \mathrm{p} \leq .001$. WWealth in terciles for Burkina Faso and in quintiles for Uganda. $¥$ First range for Burkina Faso, and second for Uganda. Notes: "Potential contraceptive users" were women not currently using a permanent contraceptive method who would consider using a newly developed contraceptive method (and in Burkina Faso, any contraceptive method) in the future. Percentages are weighted. p-values of the Rao-Scott F statistic comparing amenorrhea acceptable and amenorrhea unacceptable. Percentage distributions may not add to 100.0 because of rounding. na=not applicable. month, were pregnant or completed the women's questionnaire in a language other than French.

With regard to menstrual health practices among recently menstruating women in Burkina Faso, the space most commonly used by women to manage their menstruation was their sleeping area or another room in the household (45\%; Table 2); however, most reported the space they used was not private, safe or lockable, nor was it clean with water and soap available $(81 \%$ and $90 \%$, respectively). Fifty-nine percent used a cloth to absorb menstrual blood, and 17\% used a menstrual product, such as a tampon or menstrual pad. Only 23\% of women reported having everything they needed to manage their menstruation.

In bivariate analyses, amenorrhea acceptability was higher among women who did not use the household's main sanitation facility to manage their menstruation (59-73\% vs. 53\%). Amenorrhea acceptability also differed by the type of materials used to absorb menstrual blood, ranging from $43 \%$ among women who reported using disposable materials other than a menstrual product to $80 \%$ among those who used reusable materials other than a menstrual product or no materials. A greater proportion of women with an unmet menstrual health need than of those without were willing to accept amenorrhea (67\% vs. 45\%).

- Multivariate results. The significance of some bivariate associations changed in the multivariate models that controlled for other covariates (Table 3). In Burkina Faso, women aged 15-19 had a higher predicted probability of accepting amenorrhea than those in all older age-groups (averaged differential effects, 9-14 percentage points), although the association was only marginally significant for those aged 30-34. Women living in rural areas had a higher predicted probability than those in urban areas, and married women cohabiting with their partners had a higher predicted probability than never-married women, of amenorrhea acceptability (23 and 12 percentage points, respectively). In addition, the predicted probability of amenorrhea acceptability was six percentage points higher among current contraceptive users than among never-users. Amenorrhea acceptability was higher among women living in Mossi households than among those living in Gourmantché households (19 percentage points). Among recently menstruating women in Burkina Faso, women's predicted probabilities of amenorrhea acceptability did not differ significantly by menstrual health facility, materials or unmet need, after controlling for sociodemographic and reproductive characteristics (see Appendix Table 1).

In Uganda, women aged 15-19 had higher amenorrhea acceptability than women aged 25-29 (averaged differential effect, 12 percentage points; Table 3). Amenorrhea acceptability seemed to increase as wealth decreased, as women in the lowest wealth quintile had the highest predicted probability of accepting amenorrhea (51\%), which was significantly higher than that for women in the highest 
TABLE 2. Percentage distribution of potential contraceptive users in Burkina Faso who recently menstruated; and percentage of those who would choose a method that caused amenorrhea during use-both by menstrual health characteristics at last menses

Characteristic $(n=1,834)$

Main menstrual health facility used‡

Sleeping area/other room

Main household sanitation facility

Other sanitation facility§

Backyard/bush/no facility/other

Facility is private, safe and lockableł

No

Yes

Facility is clean with available water and soapł

No

Yes

Menstrual health materials usedt†

Cloth

Menstrual product㧊

Other disposable materials

Other reusable materials/none

Any unmet menstrual health need§§

No

Yes

Total

$* * \mathrm{p} \leq .01 . * * * \mathrm{p} \leq .001 .+\mathrm{p} \leq .10$. $\neq$ Sanitation facility or other place for changing, washing, drying or disposing of menstrual health materials. §In household, at work or school, or public facility. +†Used to collect or absorb menstrual blood. $\neq \neq$ Tampons or menstrual pad. $\S$ Answers included clean water, soap or absorbent materials; more knowledge/awareness; money; pain reliever/analgesic; and a place that is private or safe, or where one can buy clean and absorbent materials or dispose materials used. Notes: "Potential contraceptive users" were women not currently using a permanent method who would consider using a newly developed or any method in the future. "Recently menstruated" includes women who menstruated in the last 90 days and were not pregnant. Percentages are weighted. $p$-values of the Rao-Scott F statistic comparing amenorrhea acceptable and amenorrhea unacceptable. Percentage distributions may not add to 100.0 because of rounding. women described amenorrhea as desirable (seven and 10, respectively) or acceptable (10 and 14). In Uganda, however, desirable or acceptable attitudes were more common in FGDs consisting of contraceptive users (i.e., LARC or short-acting hormonal methods) than in those of nonusers (not shown).

- Reasons for attitudes about amenorrhea. In both countries, the most common reason for a desirable attitude about amenorrhea was seeing it as a way to alleviate issues experienced with standard menstrual bleeding (see Appendix Table 2). Some women saw benefits to amenorrhea because they dislike menstruation in general or experienced problematic standard bleeding. For example, one Ugandan participant said:

"Personally, I would feel good [if bleeding completely stopped with contraceptive use]... I see it is good because when I bleed, I do it excessively (heavily), and I see that if I don't bleed, I think I will be in some peace."-37-year-old past injectable user, Eastern region, Uganda

Other women found amenorrhea desirable because it permitted activities often restricted by or prohibited during menstruation. For example, although there are no biological or medical reasons for abstaining from sex during menstruation, a Burkinabè participant explained the benefit of being able to engage in sexual activity anytime while experiencing amenorrhea:

"Me, in my case, what is pleasing with the absence of your period is the fact that my husband can have sexual relations at any moment with me. But physically, I did not feel well." -27-year-old past implant user, Ouagadougou, Burkina Faso

This last quotation demonstrates another important finding: Attitudes about amenorrhea and reasons for those attitudes were multifaceted and placed within the context of other side effects, preferences and norms. We discuss this finding more below.

In Burkina Faso, another common reason for desiring or accepting amenorrhea was knowing that it was only temporary and bleeding would return after method discontinuation. In addition, the most common reason for an acceptable attitude in Burkina Faso was mistakenly equating amenorrhea with method effectiveness. These two reasons are described by one Burkinabè participant:

"For me, when one takes a method, it is to prevent a pregnancy. So, the fact that your period stops signifies that the product has truly suspended your procreation.... So, for me, when you take a method, your period stops, and when you take it out, your period comes back, and you can again [become pregnant]."-40-year-old nonuser, Southwest region, Burkina Faso

Although some in Burkina Faso, like the participant above, saw amenorrhea as an indication that the method was preventing pregnancy, a less-common alternative reason for finding amenorrhea unacceptable expressed in both countries was the concern that it was, instead, an early indication of pregnancy. 
TABLE 3. Predicted probabilities and averaged differential effects of amenorrhea acceptability among potential contraceptive users, by sociodemographic and reproductive characteristics, according to country

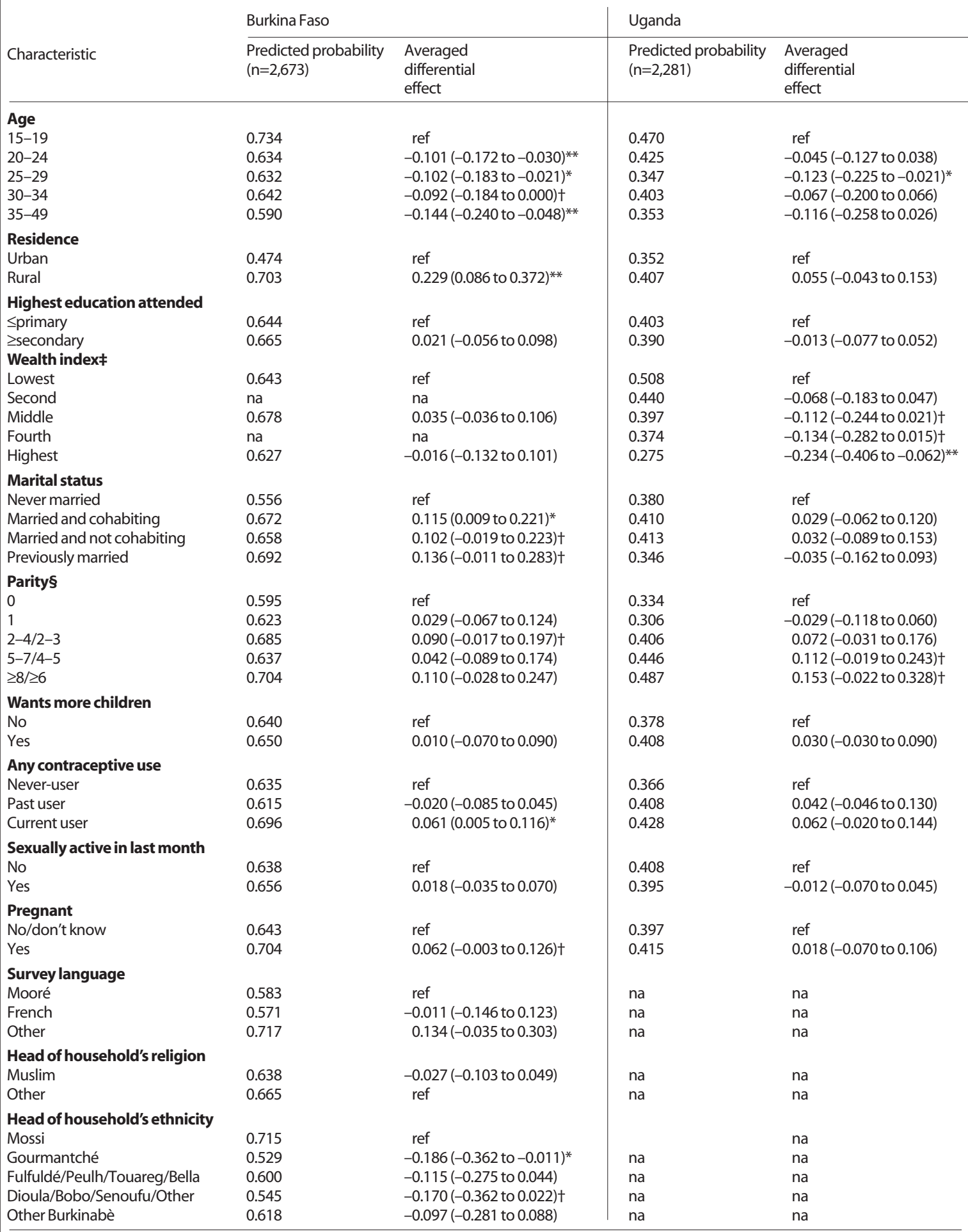

${ }^{*} p \leq .05 .{ }^{* *} p \leq .01 .+p \leq .10$. $¥$ Wealth in terciles for Burkina Faso and in quintiles for Uganda. §First range for Burkina Faso, and second for Uganda. Notes: "Potential contraceptive users" were women not currently using a permanent contraceptive method who would consider using a newly developed contraceptive method (and in Burkina Faso, any contraceptive method) in the future. Figures in parentheses are $95 \%$ confidence intervals. ref=reference group. na=not applicable.

In Uganda, provider counseling was a commonly discussed reason for finding amenorrhea desirable or acceptable. In general, the discussion was about counseling that occurred when women returned to a provider after experiencing amenorrhea, not counseling that was received with method initiation. For example, a Ugandan participant explained how providers described to her the benefit from the reduced burden of cleaning menstrual materials, also relating back to amenorrhea as a way to assuage issues of standard menstruation: 


\begin{tabular}{|c|c|c|}
\hline Characteristic & $\begin{array}{l}\text { Burkina Faso } \\
(\mathrm{n}=16)\end{array}$ & $\begin{array}{l}\text { Uganda } \\
(\mathrm{n}=30)\end{array}$ \\
\hline \multicolumn{3}{|l|}{ Type of participant } \\
\hline Nonusers & 10 & 10 \\
\hline Users & 6 & na \\
\hline LARC users & na & 10 \\
\hline SARC users & na & 10 \\
\hline Median no. of participants per FGD & 8 & 8 \\
\hline \multicolumn{3}{|l|}{ Median age of participants } \\
\hline $15-29$ & 7 & 13 \\
\hline$\geq 30$ & 9 & 17 \\
\hline \multicolumn{3}{|l|}{ Median parity of participants } \\
\hline $0-3$ & 6 & 10 \\
\hline$\geq 4$ & 10 & 20 \\
\hline \multicolumn{3}{|l|}{$\begin{array}{l}\text { Attitude about amenorrhea } \\
\text { discussed } \dagger\end{array}$} \\
\hline Desirable & 7 & 10 \\
\hline Acceptable & 10 & 14 \\
\hline Unacceptable & 14 & 26 \\
\hline \multicolumn{3}{|c|}{$\begin{array}{l}\text { +Multiple attitudes were discussed in FGDs. Desirable=e.g., good, would } \\
\text { welcome it, would be a relief or calming; Acceptable=e.g., could manage } \\
\text { it, not a problem, didn't fear it; Unacceptable=e.g., a problem, concern, not } \\
\text { good. Notes: LARC=long-acting reversible contraception (i.e., the implant } \\
\text { and IUD). SARC=short-acting reversible contraceptive (i.e., the injectable } \\
\text { and pill). na=not applicable. }\end{array}$} \\
\hline
\end{tabular}

"Me, I went to hospital to ask them about why the menstrual period has stopped, and they just asked me whether I want to wash the dirty clothes [referring to washing materials used to absorb menstrual blood]. Now I know it's not bad when [menstrual bleeding] stopped...."-37-year-old IUD user, Northern region, Uganda

Discussion about the reasons for unacceptable attitudes about amenorrhea tended to be less specific in Burkina Faso than in Uganda, and there were a few reasons only discussed in Uganda (see Appendix Table 3). In Burkina Faso, two of the most commonly discussed reasons for unacceptable attitudes about amenorrhea were its impact on general health, and general concerns and fears (see Appendix Table 2). Burkinabè women discussed how amenorrhea could cause general illness, health problems or "bring disease," as illustrated by the following participant:

"If your period does not come any more and you do not have health problems, it can be ok; but if, on the other hand, your period does not come, and you do not find your health again, this can be due to the cessation of your period and that is worrisome."-23-year-old nonuser, Southwest region, Burkina Faso

Also highlighted in this quotation is a perception discussed in some other FGDs: viewing other contraceptiveinduced health effects experienced along with amenorrhea as caused by the amenorrhea, rather than viewing all the health effects-including amenorrhea-as being caused by the contraceptive.

In another Burkinabè FGD, a participant further described this perception of amenorrhea's impact on general health: "...you do not know if the absence of your period will cause you illness or not. As long as you do not see your period, you are not at peace.... You are worried because you have the impression that your period is building up at the level of your lower stomach. And the day where it starts to flow, hum, if there is no one next to you to help you..."-28-year-old nonuser, Ouagadougou, Burkina Faso

This quotation and others below illustrate another important finding: Participants in both countries discussed the perception that menstrual blood accumulates inside of the body while experiencing amenorrhea, as well as the related concern that when menstrual bleeding does resume, it would be problematic (e.g., heavy in volume, long in duration or unpredictable). Women discussed several negative implications this type of problematic bleeding could have on their lives (e.g., trouble managing menstruation, not being able to work, not being able to have sex with their husband), especially without assistance from others

In Uganda, discussion about why amenorrhea was unacceptable tended to be more descriptive than in Burkina Faso. The three most commonly discussed reasons were related to its effect on strength and energy; the perception that women should menstruate every month; and the impact of aches or pains (see Appendix Table 2). Amenorrhea's effect on strength and energy was perceived as causing weakness, sweating and fatigue, as one 25-year-old IUD user from the Western region of Uganda explained:

Participant (P): When you fail to go on the period, this [menstrual] blood does not get out of your body. So, you feel tired all the time, you put on weight and fail even to walk short distances.

Moderator: So, those who do not go on their periods get that problem?

P: Yes, they put on a lot of weight and they can't help themselves. They feel hot, the heart beats very fast and they can't walk uphill. They can't do anything for themselves.

The view that monthly menstruation is "normal," "healthy" or "natural" was commonly discussed in Uganda. For example, one participant described how menstruating every month was viewed to be part of general religious law:

"...they say that it is a law that every woman has to release that [menstrual] blood every month. So, I don't know whether the law was in the bible or the Quran."-37year-old past injectable user, Eastern region, Uganda

Another Ugandan participant explained how monthly menstruation was taught as an indicator of a woman's health:

"...The problem is that we grew up when they were telling us every healthy woman must get periods every month. So, don't you see there is a problem spending a full year without periods? That thing that comes out [menstrual blood] where does it go?"-36-year-old nonuser, Kampala, Uganda

- Impact of amenorrhea attitudes on contraceptive decision making. We found a similar relationship between 
amenorrhea attitudes and contraceptive decision making in both countries (see Appendix Table 2). Among FGDs that discussed contraceptive decision making, desirable and acceptable attitudes about amenorrhea only resulted in low-risk contraceptive decision making (e.g., continuation). Unacceptable attitudes resulted in medium-risk (e.g., switching or choosing less-effective methods) and high-risk (e.g., nonuse or discontinuation) contraceptive decision making in some FGDs; however, in half of those FGDs, low-risk contraceptive decision making persisted despite the unacceptable attitude.

Reasons for this misalignment between attitude and decision making were discussed in several FGDs. For example, some women experiencing amenorrhea were counseled to continue contraceptive use and told pregnancy prevention was more important. Concerns often still lingered, however, as described by an injectable user in the Central region of Uganda:

"When I started going for family planning, I stopped getting my monthly period.... I went [to a health education session] and inquired about this situation. The lady who was facilitating told us that getting the monthly period is not the gist of the matter. All we should look for is the protection the methods give us...against unwanted pregnancies. She said that whatever side effect we get, we should be patient and bear it... But we are told that when you do not get the monthly period, that blood gets stuck in the uterus causing tumors, which may lead one to an operation.... This is so scary, especially to me who is a single woman without a man [to] take me to [the district] hospital for an operation if this blood went and clotted in the tubes."

In some instances, however, pregnancy prevention was seen as less important than amenorrhea, as explained by another Ugandan participant:

"For me, I want to remove it [my implant]...because I have used it for long [and I] am thinking it's the one bringing the effect of not bleeding. And we are not on good terms, with my husband. My husband got a problem [she explains later her husband is bed-ridden due to illness]. We are no longer happy, so I feel it's inconveniencing me. I'm not bleeding, and at the same time not having sex with the man."-33-year-old implant user, Eastern region, Uganda

In other FGDs, women discussed continuing use while waiting for their standard bleeding to resume over time or seeking treatment from a provider. For example, a participant in Burkina Faso described her experience taking combined oral contraceptives to treat amenorrhea caused by injectable use:

"When you now go to the health center, they tell you that [the change in bleeding] is the side effect of [the injectable]. They counsel you to take the pills so that [your period] comes as usual. When you take the pills as well, it is as like you take two methods. You have had the injection, [and] you will take the pills which can diminish the side effects of the injection, but it [is] like two projects being done at the same time in your [body]...."40-year-old past injectable user, Boucle du Mouhoun region, Burkina Faso

\section{DISCUSSION}

These findings add to the understanding of women's perspectives about contraceptive-induced amenorrhea, and they build upon the existing literature in four important ways. First, the data came from two Sub-Saharan African countries, adding to the limited data from low-income settings. Given that perceptions of amenorrhea and menstruation are dependent on social and cultural context, we cannot make assumptions about low-income countries from research focused on middle- and high-income countries. Second, the data were not limited to the context of continuous use of the pill, as is the case in much of the existing literature. Amenorrhea as a side effect of injectable, implant or IUD use is often unpredictable, and if unacceptable to the user, requires provider intervention for treatment or-for LARCs-method removal. This is quite different than continuous pill use, in which women choose to skip placebo pills to delay or prevent withdrawal bleeding, which can be discontinued anytime by the user. Third, the qualitative data allowed us to examine perspectives about contraceptive-induced amenorrhea in a more nuanced way, and to explore the stated reasons behind women's attitudes and the impact on contraceptive decision making. And fourth, our study included a multivariate analysis, which is important because we found a notable difference between our bivariate and multivariate results.

We found interesting differences and similarities between the two countries. Overall, there was a higher level of amenorrhea acceptability in Burkina Faso than in Uganda in both the quantitative and qualitative analyses. The multivariate analysis also revealed different trends in characteristics associated with women's attitudes about amenorrhea: In Burkina Faso, amenorrhea acceptability tended to be related to women's position in their reproductive life-course (i.e., age, marital status and contraceptive use) and residence, whereas in Uganda, there was an inverse relationship between wealth and amenorrhea acceptability. In addition, women's reasons for finding amenorrhea unacceptable tended to be more vague and general in Burkina Faso than the more specific reasons cited in Uganda. These differences may reflect wider use of amenorrhea-inducing methods in Uganda and, therefore, more actual experience managing real and perceived effects of amenorrhea. If true, this may also suggest a transition in perceptions about amenorrhea from higher acceptability with lower hormonal contraceptive use to decreased acceptability with increased experience and use. Alternatively, the qualitative data suggest there may be stronger norms about the need for monthly menstrual bleeding in Uganda, which could also explain lower acceptance of amenorrhea and for differing reasons. 
Despite these country differences, the relationship between attitudes and contraceptive decision making was similar in both Burkina Faso and Uganda. Desirable and acceptable attitudes were only discussed in relation to low-risk contraceptive decision making, and although some unacceptable attitudes resulted in medium- and high-risk decision making, half of the time it was still discussed in relation to low-risk decision making. This finding strongly cautions against equating method use and continuation with method satisfaction. Other research has identified a similar lack of direct alignment between women's stated attitudes and their method choice. ${ }^{34-36}$ Our findings demonstrate ways women balance competing preferences and their level of desire to prevent pregnancy. This relationship may also be related to the availability and legality of abortion services. The dynamics of this balancing and weighing of different factors is an interesting area for future research, possibly via discrete choice experiments and ranking exercises.

\section{Limitations}

The study had several limitations. First, the analysis was restricted to the target population of interest in the FHI 360 study: potential contraceptive users. Although PMA2020 data are nationally representative, our analysis sample only included women eligible for the additional study questions. Most differences between these women and women who were not eligible are to be expected of potential contraceptive users (e.g., younger, higher parity); however, in Burkina Faso, there were also differences by urban-rural residence and household ethnicity. In the qualitative sample, women opposed to contraception or currently trying to get pregnant were excluded. Although these restrictions may limit the generalizability and transferability of our results, the perspectives of these potential contraceptive users are of great interest. Ways to address the need to prevent unintended pregnancy among women who do not intend to use a contraceptive is an important area for further study.

A related second limitation is the imperfect ability of people to accurately predict their own behavior. Particularly for women with no experience with contraceptive-induced amenorrhea, and possibly even for women with limited contraceptive experience more broadly, it may be difficult to predict attitudes about amenorrhea and how they might affect contraceptive decision making.

With regard to how our results extend to estimating future use of the current amenorrhea-inducing methods, it is important to note that neither our quantitative nor qualitative data explicitly explored issues related to the predictability of amenorrhea and return to menstruation after method discontinuation. This distinction is especially relevant for longer acting methods that may cause amenorrhea, other menstrual changes, or no menstrual changes. These issues, therefore, warrant future study.

\section{CONCLUSIONS}

Our results have implications for provider counseling and for social and behavior change communication messaging about contraceptive-induced amenorrhea. First, we found provider counseling had a role in women's acceptability of amenorrhea, most notably in Uganda; however, such counseling appeared to be lacking before women began a method. Better provider tools for counseling about contraceptive-induced menstrual bleeding changes may improve this deficit. ${ }^{37}$ In addition, although reassurance from a provider may adequately alleviate some women's concerns about amenorrhea, it should not be at the expense of fully acknowledging those concerns and, if desired, a woman's decision to discontinue or switch methods.

Second, a common reason in both countries for desiring amenorrhea was its potential to alleviate problems women experienced with their standard menstrual bleeding; however, it should be noted that we only found menstrual health practices to be significantly related to amenorrhea acceptability in Burkina Faso in our bivariate analysis, not our multivariate analysis. Counseling about and promotion of these types of "lifestyle" or "wellness" benefits may help improve amenorrhea acceptability, but it is important to note many of these benefits are only realized when amenorrhea is predictable and consistent, which is not always the case with contraceptive-induced amenorrhea, especially with current longer acting methods. ${ }^{5,8}$ Efforts in contraceptive counseling about amenorrhea-especially if temporary or unpredictable-and other menstrual changes could be aided by integrating the provision of menstrual products within family planning service delivery. In addition, marketing or counseling for amenorrhea-inducing methods should not be done in ways that further promote menstrual stigma or in place of addressing larger unmet menstrual health needs that may underpin the reason some women may seek to avoid menstruating with contraception.

Third, we found a notable number of misconceptions about how contraceptives work, why amenorrhea happens and menstruation in general. Addressing these misperceptions with education and counseling may improve some women's attitudes about contraceptive-induced amenorrhea. In addition, improving education and understanding on these topics will likely produce more informed contraceptive users.

Finally, our results are useful for guiding the development of new contraceptive methods. They point to a market for new methods that cause amenorrhea-ideally predictably-in low-income countries where some women find amenorrhea acceptable and even desirable. However, the findings also support the need for other new methods to provide a range of options for women who do not wish to become amenorrhoeic.

Overall, efforts to improve provider counseling and social and behavior change communication messaging 
about amenorrhea with existing methods and the development of improved new methods with deference toward women's expressed preferences about contraceptive-induced amenorrhea will better meet the needs of women and couples, so they can exercise their human right to determine if and when they choose to have children.

\section{REFERENCES}

1. Sedgh G, Ashford LS and Hussain R, Unmet Need for Contraception in Developing Countries: Examining Women's Reasons for Not Using a Method, New York: Guttmacher Institute, 2016, https://www.guttmacher.org/report/ unmet-need-for-contraception-in-developing-countries

2. Darroch JE, Sedgh G and Ball H, Contraceptive Technologies: Responding to Women's Needs, New York: Guttmacher Institute, 2011, https://www.guttmacher.org/sites/default/files/report_pdf/ contraceptive-technologies.pdf

3. Sedgh $G$ and Hussain R, Reasons for contraceptive nonuse among women having unmet need for contraception in developing countries, Studies in Family Planning, 2014, 45(2):151-169, http:// dx.doi.org/10.1111/j.1728-4465.2014.00382.x.

4. Demographic and Health Survey Program, DHS STATcompiler, 2016, http://www.statcompiler.com.

5. Hubacher D et al., Menstrual pattern changes from levonorgestrel subdermal implants and DMPA: systematic review and evidencebased comparisons, Contraception, 2009, 80(2):113-118, http:// dx.doi.org/10.1016/j.contraception.2009.02.008.

6. Hickey $M$ and d'Arcangues $C$, Vaginal bleeding disturbances and implantable contraceptives, Contraception, 2002, 65(1):75-84, http://dx.doi.org/10.1016/S0010-7824(01)00292-X

7. Bachmann $G$ and Korner P, Bleeding patterns associated with non-oral hormonal contraceptives: a review of the literature, Contraception, 2009, 79(4):247-258, http://dx.doi.org/10.1016/j. contraception.2008.10.012.

8. Sergison JE et al., Levonorgestrel intrauterine system associated amenorrhea: a systematic review and metaanalysis, American Journal of Obstetrics \& Gynecology, 2019, 220(5):440-448.e8, http://dx.doi. org/10.1016/j.ajog.2018.12.008.

9. Polis CB, Hussain R and Berry A, There might be blood: a scoping review on women's responses to contraceptive-induced menstrual bleeding changes, Reproductive Health, 2018, 15(1):114, http:// dx.doi.org/10.1186/s12978-018-0561-0.

10. Association of Reproductive Health Professionals (ARHP), Menstruation and Menstrual Suppression Survey, Washington, DC, USA: ARHP, 2006, https://www.arhp. org/publications-and-resources/studies-and-surveys/ menstruation-and-menstrual-suppression-survey.

11. Makuch MY et al., Opinion and experience of Brazilian women regarding menstrual bleeding and use of combined oral contraceptives, International Journal of Gynaecology $\&$ Obstetrics, 2012, 117(1):5-9, http://dx.doi.org/10.1016/j. ijgo.2011.11.018

12. Rose JG, Chrisler JC and Couture S, Young women's attitudes toward continuous use of oral contraceptives: the effect of priming positive attitudes toward menstruation on women's willingness to suppress menstruation, Health Care for Women International, 2008, 29(7):688-701, http://dx.doi org/10.1080/07399330802188925.

13. Clark LR et al., Menstrual irregularity from hormonal contraception: a cause of reproductive health concerns in minority adolescent young women, Contraception, 2006, 74(3):214-219, http://dx.doi.org/10.1016/j.contraception.2006.03.026.

14. Estanislau do Amaral MC et al., Menstruation and amenorrhea: opinion of Brazilian women, Contraception, 2005, 72(2):157-161, http://dx.doi.org/10.1016/j.contraception.2005.02.013.
15. Edelman A et al., Acceptability of contraceptive-induced amenorrhea in a racially diverse group of US women, Contraception, 2007, 75(6):450-453, http://dx.doi.org/10.1016/j. contraception.2007.02.005

16. Newton V and Hoggart L, Menstruation and contraception: social and cultural issues on young women's decision making, Contraception, 2015, 92(4):403, http://dx.doi.org/10.1016/j. contraception.2015.06.193

17. World Health Organization, A cross-cultural study of menstruation: implications for contraceptive development and use, Studies in Family Planning, 1981, 12(1):3-16, http://dx.doi. org/10.2307/1965859.

18. Tolley EE et al., Preferences for a potential longer-acting injectable contraceptive: perspectives from women, providers, and policy makers in Kenya and Rwanda, Global Health, Science and Practice, 2014, 2(2):182-194, http://dx.doi.org/10.9745/ GHSP-D-13-00147.

19. Das M, Socio-cultural aspects of menstruation: an anthropological purview, Eastern Anthropologist, 2008, $61(2): 227-240$

20. Glasier AF et al., Amenorrhea associated with contraception-an international study on acceptability, Contraception, 2003, 67(1):1-8, http://dx.doi.org/10.1016/S0010-7824(02)00474-2.

21. den Tonkelaar I and Oddens BJ, Preferred frequency and characteristics of menstrual bleeding in relation to reproductive status, oral contraceptive use, and hormone replacement therapy use, Contraception, 1999, 59(6):357-362, http://www.ncbi.nlm.nih. gov/pubmed/10518229.

22. Hardy E et al., Association between characteristics of current menses and preference for induced amenorrhea, Contraception, 2009, 80(3):266-269, http://dx.doi.org/10.1016/j contraception.2009.06.010

23. Snow R et al., Women's responses to menses and nonbleeding intervals in the USA, Brazil and Germany, Contraception, 2007, 76(1):23-29, http://dx.doi.org/10.1016/j. contraception.2007.03.008

24. Callahan RL et al., Potential user interest in new long-acting contraceptives: results from a mixed methods study in Burkina Faso and Uganda, PLOS ONE, 2019, 14(5):e0217333, http://dx.doi. org/10.1371/journal.pone.0217333.

25. Brunie A et al., Developing acceptable contraceptive methods: mixed-method findings on preferred method characteristics from Burkina Faso and Uganda, Gates Open Research, 2019, 3:1205, http://dx.doi.org/10.12688/gatesopenres.12953.2.

26. Performance Monitoring and Accountability 2020 (PMA2020), PMA2016/Burkina Faso Round 4 Snapshot of Indicators, 2016, https:// www.pmadata.org/countries/burkina-faso/burkina-faso-indicators/ pma2016burkina-faso-round-4-indicators.

27. Performance Monitoring and Accountability 2020 (PMA2020), PMA2016/Uganda Round 4 Snapshot of Indicators, 2016, https:// www.pmadata.org/countries/uganda/uganda-indicators/ pma2016uganda-round-4-indicators

28. Performance Monitoring and Accountability 2020 (PMA2020), PMA2016/Burkina Faso Round 4 Family Planning Brief, Ouagadougou, 2017, https://www.pmadata.org/sites/default/files/data_product_ results/PMA2020-Burkina-R4-FP-Brief-EN.pdf

29. Performance Monitoring and Accountability 2020 (PMA2020), PMA2016/Uganda Round 4 Family Planning Brief, 2018, https://www pmadata.org/sites/default/files/data_product_results/PMA2020Uganda-R4-FP-brief.pdf.

30. Guttmacher Institute, Abortion in Burkina Faso, Fact Sheet, 2014, https://www.guttmacher.org/fact-sheet/abortion-burkina-faso.

31. Guttmacher Institute, Abortion and postabortion care in Uganda, Fact Sheet, 2017, https://www.guttmacher.org/fact-sheet/ abortion-and-postabortion-care-uganda.

32. Sedgh G et al., Abortion incidence between 1990 and 2014: global, regional, and subregional levels and trends, 
Lancet, 2016, 388(10041):258-267, http://dx.doi.org/10.1016/ S0140-6736(16)30380-4

33. Tolley E et al., The impact of menstrual side effects on contraceptive discontinuation: findings from a longitudinal study in Cairo, Egypt, International Family Planning Perspectives, 2005, 31(1):15-23, http://dx.doi.org/10.1363/3101505.

34. Marshall C et al., Young women's contraceptive decision making: Do preferences for contraceptive attributes align with method choice? Perspectives on Sexual and Reproductive Health, 2016, 48(3):119-127, http://doi.wiley.com/10.1363/48e10116. doi:10.1363/48e10116

35. He K et al., Women's contraceptive preference-use mismatch, Journal of Women's Health, 2017, 6(6):692-701, http://online. liebertpub.com/doi/10.1089/jwh.2016.5807.

36. Gomez A and Clark J, Method mismatch: discrepancies between young women's contraceptive preferences and current method choice, Contraception, 2013, 88(3):459-460, http://dx.doi. org/10.1016/j.contraception.2013.05.112.

37. Rademacher KH et al., Menstrual bleeding changes are NORMAL: proposed counseling tool to address common reasons for non-use and discontinuation of contraception, Global Health, Science and Practice, 2018, 6(3):603-610, http://dx.doi.org/10.9745/ GHSP-D-18-00093.

\section{RESUMEN}

Contexto: Las preocupaciones de las mujeres acerca de los cambios menstruales inducidos por los anticonceptivos pueden conducir a la interrupción y el abandono del método, lo cual contribuye a la necesidad insatisfecha de anticoncepción. La investigación sobre la percepción de la amenorrea por parte de las mujeres sobre los métodos de acción más prolongada y en los países de bajos ingresos es limitada.

Métodos: Los datos provienen de encuestas representativas de hogares a nivel nacional y discusiones de grupos focales con mujeres en edad reproductiva realizadas en Burkina Faso y Uganda entre 2016 y 2017. Se utilizaron tabulaciones cruzadas bivariadas y análisis de regresión logística multivariada para examinar las características sociodemográficas y reproductivas asociadas con las actitudes de las mujeres con respecto a la amenorrea inducida por anticonceptivos $(n=2,673$ para Burkina Faso y 2,281 para Uganda); también se examinaron los determinantes de la salud menstrual en Burkina Faso. Se analizaron datos cualitativos de discusiones de los grupos focales para comprender las razones que determinan las actitudes de las mujeres y cómo influyen en la toma de decisiones sobre anticonceptivos.

Resultados: El 65\% de las mujeres en Burkina Faso y el $40 \%$ en Uganda informaron que elegirían un método que les causara amenorrea durante su uso. En Burkina Faso, la probabilidad predicha de aceptar la amenorrea fue mayor para las mujeres de 15 a 19 años (en comparación con las mujeres mayores), que vivían en zonas rurales, que estaban casadas y cohabitaban (en comparación con las que nunca se habian casado), que actualmente usaban un método anticonceptivo (en comparación con las mujeres que nunca lo habian usado) y de hogares Mossi (en comparación con Gourmantché). Las prácticas de salud menstrual no se asociaron con la aceptabilidad de la amenorrea. En Uganda, las mujeres menos ricas tuvieron la probabilidad más alta de aceptar amenorrea (51\%). El análisis cualitativo reveló una variedad de razones con respecto a las actitudes de las mujeres sobre la amenorrea y las diferencias por país, pero la relación entre estas actitudes y la toma de decisiones sobre anticonceptivos fue similar en todos los países.

Conclusiones: El abordaje de los conceptos erróneos sobre la anticoncepción y la menstruación podría resultar en una toma de decisiones más informada sobre los métodos anticonceptivos.

\section{RESUMEN}

Contexte: Les inquiétudes des femmes à l'égard des changements menstruels induits par la contraception peuvent conduire à l'arrêt ou à la non-utilisation de la méthode et contribuer ainsi au besoin non satisfait de contraception. La recherche sur les perceptions des femmes de l'aménorrhée liée aux méthodes à durée d'action prolongée et dans les pays à revenu faible est limitée.

Méthodes: Les données sont extraites d'enquêtes de ménage nationalement représentatives et de discussions de groupe avec des femmes en âge de procréer, menées au Burkina Faso et en Ouganda en 2016-2017. Les caractéristiques sociodémographiques et reproductives associées aux attitudes des femmes concernant l'aménorrhée induite par la contraception ( $n=2673$ pour le Burkina Faso et 2281 pour l'Ouganda) ont été examinées en tableaux croisés bivariés et par analyses de régression logistique multivariée. Les déterminants de la santé menstruelle ont aussi été examinés pour le Burkina Faso. L'analyse des données qualitatives obtenues des discussions de groupe a permis de cerner les raisons à la base des attitudes des femmes et leur influence sur les décisions contraceptives prises.

Résultats: Soixante-cinq pour cent des femmes burkinabè et $40 \%$ de leurs homologues ougandaises ont déclaré qu'elles choisiraient une méthode dont la pratique causerait l'aménorrhée. Au Burkina Faso, la probabilité prédite d'acceptation de l'aménorrhée s'est avérée supérieure pour les femmes âgées de 15 à 19 ans (par rapport à leurs aînées), vivant en milieu rural, mariées ou en union (par rapport à celles qui n'avaient jamais été mariées), pratiquant actuellement la contraception (par rapport à celles qui ne l'avaient jamais pratiquée) et d'origine Mossi (par rapport à Gourmantché). Les pratiques de santé menstruelle n'étaient pas associées à l'acceptabilité de l'aménorrhée. En Ouganda, les femmes les moins riches sont associées à la plus haute probabilité prédite d'acceptation de l'aménorrhée (51\%). L'analyse qualitative a révélé diverses raisons à la base des attitudes des femmes à l'égard de l'aménorrhée ainsi que certaines différences suivant le pays, mais la relation entre ces attitudes et la décision contraceptive s'est avérée similaire dans les deux pays.

Conclusions: La résolution des idées fausses concernant la contraception et la menstruation pourrait conduire à une prise de décision mieux éclairée dans le choix des méthodes. 


\section{Acknowledgments}

This secondary analysis was part of the first author's doctoral dissertation research. The Cynthia H. Cassell Doctoral Dissertation Award in Maternal and Child Health at UNC funded the translation of portions of the focus group discussion transcripts from Burkina Faso by Amelia Maytan-Joneydi for this secondary analysis. The authors thank the FHI 360 study country investigators, Georges Guiella and Madeleine Wayack-Pambè in Burkina Faso, and Frederick Makumbi and Simon Kibira in Uganda; the PMA2020 team at the Johns Hopkins Bloomberg School of Public Health, especially
Linnea Zimmerman; and Claire Brennan, Dominika Fiolna, Saskia Guerrier, Anna Lawton, and Seth Zissette for support for the qualitative analysis. The FHI 360 study was funded by the Bill and Melinda Gates Foundation (OPP1055878, the Contraceptive Technology Innovation Initiative). The funders had no role in data analysis, decision to publish or preparation of the manuscript.

Author contact: amackenzie@fhi360.org 
APPENDIX TABLE 1. Predicted probabilities and averaged differential effects of amenorrhea acceptability among women in Burkina Faso aged 15-49 who were potential contraceptive users and who recently menstruated, by sociodemographic, reproductive and menstrual health characteristics

Characteristic

Predicted

Age

$15-19$

$20-24$

$25-29$

$30-34$

$35-49$

Residence

Urban

Rural

Averaged differential effect

Highest education attended

sprimary

$\geq$ secondary

Wealth index

Lowest

Middle

Highest

Marital status

Never married

Married and cohabiting

Married and not cohabiting

Previously married

Parity

0

1

2-4

5-7

$\geq 8$

$0.675 \quad$ ref

0.569

0.589

0.616

0.607

0.470

0.679

0.613

0.629

0.619

0.646

0.595

0.568

0.640

0.588

0.680

0.588

0.614

0.636

0.602

0.711

ants more children

0.595

Yes

0.595
0.622

Any contraceptive use

0.588

Never-user

0.604

0.675

Current user

0.607

No

0.607
0.624

Survey language

Mooré

French

Other

0.583

0.521

0.679

0.606

Muslim

0.635

Other

0.676

0.525

0.565

0.516

0.593

(

Other Burkinabè

Main menstrual health facility used‡

Sleeping area/other room

0.594

0.626

0.670

0.560

Other sanitation facility

Backyard/bush/no facility/other

Facility is private, safe and lockable‡

No

0.622

0.599

$.06(-0.191$ to -0.020$)$

$-0.086(-0.180$ to 0.009$) \dagger$

$-0.059(-0.158$ to 0.041$)$

-0.068 ( -0.193 to 0.058$)$

ref

$0.208(0.064-0.353)^{* *}$

ref

$0.016(-0.072$ to 0.105$)$

0.028 (-0.057 to 0.112$)$

-0.024 ( -0.136 to 0.089 )

ref

$0.073(-0.037$ to 0.182$)$

0.021 ( -0.104 to 0.146$)$

0.112 (-0.044 to 0.269 )

ref

0.026 (-0.096 to 0.148$)$ 0.048 (-0.081 to 0.177$)$

0.014 ( -0.136 to 0.164$)$

0.123 (-0.043 to 0.289 )

ref

$0.027(-0.068$ to 0.122$)$

ref

$0.016(-0.071$ to 0.102$)$

$0.086(0.028-0.145)^{* * *}$

ref

0.018 (-0.049 to 0.084$)$

ref

$-0.062(-0.191$ to 0.068$)$

0.096 (-0.079 to 0.272 )

$-0.030(-0.104$ to 0.044$)$

ref

$-0.151(-0.336$ to 0.034$)$

-0.111 ( -0.273 to 0.050$)$

$-0.161(-0.326$ to 0.005$) \dagger$

$-0.084(-0.278$ to 0.111$)$

ref

$0.032(-0.041$ to 0.105$)$

$0.076(-0.018$ to 0.169$)$

-0.034 ( -0.147 to 0.078 )

ref

$-0.023(-0.133$ to 0.087$)$

0.613

0.653

ref

$0.040(-0.100$ to 0.180$)$

ref

0.622

0.664

0.548

$0.042(-0.044$ to 0.128$)$

-0.073 (-0.175 to 0.028$)$

-0.001 ( -0.108 to 0.105 )

Other reusable material/none

0.621

0.579

$0.050(-0.022$ to 0.121$)$

Yes

0.629

${ }^{*} p \leq 05$. ** $p \leq .01 .+p \leq .10$. $\neq$ Sanitation facility or other place for changing, washing, drying or disposing of menstrual health materials. \$In household, at work or school, or public facility. +tUsed to collect or absorb menstrual blood. \#+Tampons or menstrual pad.
§§Answers included clean water, soap or absorbent materials; more knowledge/awareness; money; pain reliever/analgesic; and a place that is private or safe, or where one can buy clean and absorbent materials or dispose materials used. Notes: "Potential contraceptive users" were women not currently using a permanent method who would consider using a newly developed or any method in the future. "Recently menstruated" includes women who menstruated in the last 90 days and were not pregnant. Figures in parentheses are $95 \%$ confidence intervals. ref=reference group. 
APPENDIX TABLE 2. Number of focus group discussions in which reasons for amenorrhea attitudes and the impact on contraceptive decision making were discussed, by country

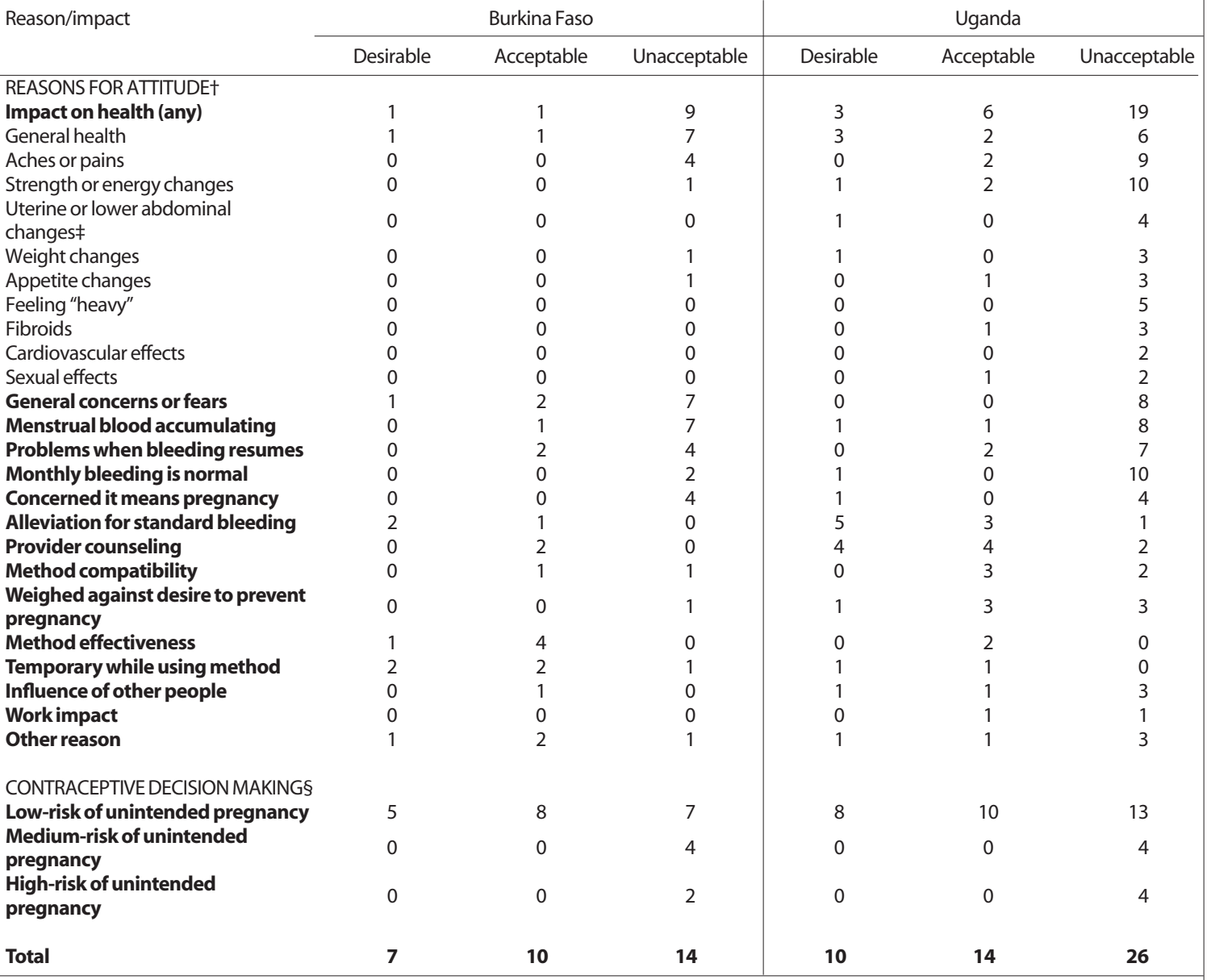

†The following reasons were only cited in one FGD per country or one FGD total: impact on partner, and among impacts on health, bloating or swelling, impact on fertility, or other health reason. \#Uterine or lower abdominal changes (i.e., pain, "movement"/cramping, or bloating/swelling) includes those in which women specifically mentioned the uterus or lower abdomen. When not explicitly about the uterus, stomach pain, aches, or other problems were included under general "aches or pains", and stomach or body bloating, or swelling was included under general "bloating or swelling." §Low-risk: continue using, would use, or not a barrier to use; Medium-risk: switching methods, pausing method, choosing less effective method, consider discontinuing or switching; High-risk: not using, discontinuing, would not use. Notes: Multiple attitudes, reasons and contraceptive use or decision-making impacts were discussed in FGDs. FGD=focus group discussion. 


\begin{tabular}{|c|c|c|}
\hline Reason/impact & Burkina Faso & Uganda \\
\hline \multicolumn{3}{|l|}{ REASONS FOR ATTITUDE† } \\
\hline Impact on health (any) & 12 & 26 \\
\hline General health & 10 & 14 \\
\hline Aches or pains & 6 & 17 \\
\hline Strength or energy changes & 1 & 12 \\
\hline $\begin{array}{l}\text { Uterine or lower abdominal } \\
\text { changes } \neq\end{array}$ & 0 & 8 \\
\hline Bloating or swelling & 2 & 4 \\
\hline Weight changes & 2 & 4 \\
\hline Appetite changes & 1 & 5 \\
\hline Feeling "heavy" & 1 & 5 \\
\hline Fibroids & 1 & 4 \\
\hline Cardiovascular effects & 0 & 5 \\
\hline Impact on fertility & 2 & 2 \\
\hline Sexual effects & 0 & 3 \\
\hline Other health reason & 0 & 1 \\
\hline General concerns or fears & 9 & 9 \\
\hline Menstrual blood is accumulating & 9 & 9 \\
\hline \multirow{2}{*}{$\begin{array}{l}\text { Problems when bleeding resumes } \\
\text { Monthly bleeding is normal }\end{array}$} & 4 & 12 \\
\hline & 2 & 14 \\
\hline Concerned it means pregnancy & 6 & 8 \\
\hline Alleviation for standard bleeding & 3 & 9 \\
\hline \multirow{2}{*}{$\begin{array}{l}\text { Provider counseling } \\
\text { Method compatibility }\end{array}$} & 3 & 8 \\
\hline & 4 & 6 \\
\hline $\begin{array}{l}\text { Weighed against desire to prevent } \\
\text { pregnancy }\end{array}$ & 2 & 7 \\
\hline Method effectiveness & 6 & 2 \\
\hline \multirow{2}{*}{$\begin{array}{l}\text { Temporary while using method } \\
\text { Influence of other people }\end{array}$} & 4 & 2 \\
\hline & 1 & 4 \\
\hline \multirow{2}{*}{$\begin{array}{l}\text { Partner impact } \\
\text { Work impact }\end{array}$} & 2 & 2 \\
\hline & 0 & 2 \\
\hline Other reason & 5 & 5 \\
\hline \multicolumn{3}{|l|}{ CONTRACEPTIVEDECISION MAKING§ } \\
\hline \multirow{2}{*}{$\begin{array}{l}\text { Low-risk of unintended pregnancy } \\
\text { Medium-risk of unintended } \\
\text { pregnancy }\end{array}$} & 13 & 24 \\
\hline & 5 & 8 \\
\hline $\begin{array}{l}\text { High-risk of unintended } \\
\text { pregnancy }\end{array}$ & 6 & 7 \\
\hline Total & 16 & 30 \\
\hline \multicolumn{3}{|c|}{$\begin{array}{l}\text { †Te following reasons were only cited in one FGD per country or one FGD } \\
\text { total: impact on partner, and among impacts on health, bloating or swelling, } \\
\text { impact on fertility, or other health reason. fUterine or lower abdominal changes } \\
\text { (i.e., pain, "movement"/cramping, or bloating/swelling) includes those in } \\
\text { which women specifically mentioned the uterus or lower abdomen. When } \\
\text { not explicitly about the uterus, stomach pain, aches, or other problems were } \\
\text { included under general "aches or pains", and stomach or body bloating, or } \\
\text { swelling was included under general "bloating or swelling." §Low-risk: continue } \\
\text { using, would use, or not a barrier to use; Medium-risk: switching methods, } \\
\text { pausing method, choosing less effective method, consider discontinuing or } \\
\text { switching; High-risk: not using, discontinuing, would not use. Notes: Multiple } \\
\text { attitudes, reasons and contraceptive use or decision-making impacts were } \\
\text { discussed in FGDs. This table includes discussion of amenorrhea where the } \\
\text { attitude was not explicitly stated. FGD=focus group discussion. }\end{array}$} \\
\hline
\end{tabular}

